\title{
Genomic and Proteomic Comparative Analysis of SARS-CoV-2 versus SARS-CoV-GD01
}

\author{
Abdallah A. Hassanin \\ Zagazig University Faculty of Agriculture \\ EL-Sayed I. Mahgoub \\ Zagazig University Faculty of Agriculture \\ Basel Sitohy \\ Umea Universitet \\ Mahmoud Sitohy ( $\nabla$ mzsitohy@zu.edu.eg ) \\ Zagazig University Faculty of Agriculture https://orcid.org/0000-0001-6623-4860
}

\section{Research}

Keywords: SARS-CoV-GD01, SARS-CoV2, COVID-19, Genomic, Proteomic, Coronavirus

Posted Date: June 17th, 2020

DOl: https://doi.org/10.21203/rs.3.rs-32189/v2

License: (c) (i) This work is licensed under a Creative Commons Attribution 4.0 International License. Read Full License 


\section{Abstract}

The authors have withdrawn this preprint due to author disagreement. 